\title{
Artigo/Article
}

\section{Ocorrência de Cryptococcus neoformans em excretas de pombos na Cidade de Pelotas, Estado do Rio Grande do Sul}

\author{
Occurrence of Cryptococcus neoformans in pigeon excrement in the City of Pelotas, State of \\ Rio Grande do Sul
}

\author{
Renata Osório de Faria ${ }^{1}$, Patrícia da Silva Nascente ${ }^{2}$, Ana Raquel Mano Meinerz ${ }^{2}$, Marlete Brum Cleff ${ }^{3}$, \\ Tatiana de Avila Antunes ${ }^{1}$, Érica da Silva Silveira ${ }^{4}$, Márcia de Oliveira Nobre $^{3}$, Mário Carlos Araújo Meireles ${ }^{5}$ \\ e João Roberto Braga de Mello ${ }^{6}$
}

\section{RESUMO}

Introdução: O Cryptococcus neoformansé uma levedura capsulada, agente etiológico da criptococose em humanos e animais, encontrado em fontes ambientais, incluindo excretas de pombos, é uma importante causa de mortalidade em indivíduos imunodeprimidos em todo o mundo. Métodos: Com o objetivo de verificar a ocorrência do Cryptococcus neoformans, em excretas de pombos, na Cidade de Pelotas, pesquisou-se 70 ambientes, incluindo prédios, praças e locais ao ar livre, da Cidade de Pelotas, RS. Após a coleta, os excrementos foram adicionados de salina com cloranfenicol, homogeneizados, semeados em ágar Sabouraud com cloranfenicol e ágar Níger e incubados a $32^{\circ} \mathrm{C}$. Para identificação, realizou-se exame direto, prova da fenoloxidase, urease, assimilação de carboidratos e cultura em meio CGB. Resultados: Dos locais estudados $\left(\mathrm{n}^{\circ}=70\right)$, em $26(37,1 \%)$ havia excretas de pombos. Estes lugares foram representados por prédios históricos $\left(\mathrm{n}^{\mathrm{o}}=8\right)$, torre de igreja $\left(\mathrm{n}^{\mathrm{o}}=1\right)$, engenhos e armazéns de arroz $\left(n^{\circ}=7\right)$, praça $\left(n^{\circ}=1\right)$ e locais ao ar livre $\left(n^{\circ}=9\right)$, o isolamento de Cryptococcus neoformans ocorreu em $26,9 \%\left(n^{\circ}=7 / 26\right)$, destes locais. Conclusões: O estudo chama a atenção, para o isolamento do fungo em áreas urbanas, que apresentavam grande acúmulo de excretas, indicando um risco para a saúde pública, especialmente para indivíduos imunocomprometidos.

Palavras-chaves: Cryptococcus neoformans. Excretas. Pombos. Ambiente. Urbano.

\begin{abstract}
Introduction: Cryptococcus neoformans is an encapsulated yeast and is the etiological agent for human and animal cryptococcosis. It is found in sources within the environment, including pigeon excrement, and is an important cause of mortality among immunocompromised individuals worldwide. Methods: Seventy different environments in the city of Pelotas, Rio Grande do Sul, were surveyed for the purpose of investigating Cryptococcus neoformans occurrences in pigeon excreta. The environments included buildings, public squares and outdoor locations in the city. After collection, chloramphenicol saline solution was added to the excreta, which were then homogenized and seeded onto Sabouraud agar with chloramphenicol and onto Niger agar, and incubated at $32^{\circ} \mathrm{C}$. Identification was performed by direct examination and by means of the phenoloxidase and urease tests, carbohydrate assimilation and culturing in CGB medium. Results: Out of the sites investigated $(\mathrm{n}=70), 26(37.1 \%)$ of them contained pigeon excreta. These included historical buildings $(\mathrm{n}=8)$, a church tower $(\mathrm{n}=1)$, rice mills and warehouses $(\mathrm{n}=7)$, a public square $(\mathrm{n}=1)$ and outdoor locations $(\mathrm{n}=9)$. Cryptococcus neoformans was isolated from $26.9 \%(n=7 / 26)$ of these locations. Conclusions: This study draws attention to isolation of this fungus in urban areas that present large accumulations of pigeon excrement. This represents a risk to public health, especially for immunocompromised individuals.
\end{abstract}

Key-words: Cryptococcus neoformans. Excrement. Pigeons. Environment. Urban.

1. Programa de Pós-Graduação em Ciências Veterinárias, Faculdade de Veterinária, Universidade Federal do Rio Grande do Sul. Porto Alegre, RS. 2. Departamento de Microbiologia e Parasitologia, Instituto de Biologia, Universidade Federal de Pelotas. Pelotas, RS. 3. Departamento de Clínica Veterinária, Faculdade de Veterinária, Universidade Federal de Pelotas, Pelotas, RS. 4. Departamento de Microbiologia, Fundação Universidade Federal de Rio Grande. Rio Grande, RS. 5. Setor de Micologia, Laboratório de Doenças Infecciosas, Departamento de Veterinária Preventiva, Faculdade de Veterinária, Universidade Federal de Pelotas, Pelotas, RS. 6. Departamento de Farmacologia, Instituto de Ciências Básicas da Saúde, Universidade Federal do Rio Grande do Sul, Porto Alegre, RS.

Endereço para correspondência: Dra Renata Osório de Faria. Rua Uruguai 1152, 96010-630 Pelotas, RS. Tel: 5553 3275-7496

e-mail: renataosorio@ig.com.br

Recebido para publicação em 24/08/2009

Aceito em 20/01/2010

\section{INTRODUÇÃO}

O gênero Cryptococcus comporta mais de 38 espécies, duas das quaissão consideradas potencialmente patogênicas, Cryptococcus neoformans e Cryptococcus gatti ${ }^{1,2,3}$, agentes etiológicos da criptococose, uma micose grave que tem tido uma crescente importância nas últimas décadas ${ }^{1,4}$. A infecção causada por Cryptococcus neoformans ocorre preferencialmente em indivíduos imunocomprometidos, enquanto Cryptococcus gattii também acomete indivíduos imunocompetentes ${ }^{5}$.

A fonte ambiental do Cryptococcus gattii está associada a eucaliptos e material vegetal em decomposição ${ }^{5}$. No Brasil, o Cryptococcus gattii foi também encontrado em cássia rosa (Cassia grandis), ficus (Ficus microcarpa) e outras árvores nativas, evidenciando outros habitats naturais para essa espécie ${ }^{5}$.

Cryptococcus neoformans no ambiente é principalmente encontrado em excretas de aves, especialmente de pombos (Columba livia), e o ecossistema urbano tem se tornado propício para permanência destas aves, proporcionando acúmulo de excretas, que é considerada a fonte de infecção mais importante para homens e animais. Aliado a este fato, a população de pombos domésticos tem aumentado significativamente em diversas partes do mundo, inclusive no Brasil, tornandose um problema ambiental e de saúde pública ${ }^{6-8}$. Esta espécie tem o hábito de viverem em grupos, abrigando-se e construindo seus ninhos em locais altos como prédios, torres, sótãos, beirais de janelas, entre outros. Estas aves alimentam-se preferencialmente de grãos, mas aproveitam bem os restos de alimentos e lixo encontrados em locais públicos como, por exemplo, praças e parques ${ }^{9,10}$.

Pelotas está localizada no sul do Rio Grande do Sul, sendo considerada um dos mais ricos patrimônios culturais e arquitetônicos do Brasil. Esta cidade apresenta grande número de prédios 
históricos, igrejas, praças públicas, além de possuir inúmeros engenhos de arroz e armazéns de grãos. Estes ambientes são considerados um ótimo habitat para habitação e reprodução de pombos domésticos. Assim, o objetivo deste trabalho foi verificar a ocorrência de Cryptococcus neoformans em excretas de pombos em área urbana na Cidade de Pelotas, RS, Brasil.

\section{MÉTODOS}

Para a realização do estudo, selecionou-se 70 locais da Cidade de Pelotas, por terem a presença de pombos continuamente. Os locais selecionados foram engenhos e armazéns de grãos $\left(\mathrm{n}^{\circ}=7\right)$, praças $\left(\mathrm{n}^{\mathrm{o}}=14\right)$ e ruas $\left(\mathrm{n}^{\mathrm{o}}=9\right)$, prédios históricos $\left(\mathrm{n}^{\mathrm{0}}=33\right)$ e torres de igrejas $\left(\mathrm{n}^{\mathrm{0}}=7\right)$, por apresentarem construções ricas em ornamentos e detalhes esculturais que servem de abrigo ou como locais de alimentação e reprodução dos pombos.

Estes locais foram vistoriados e classificados conforme a quantidade de excretas no local, pelo seguinte escore: 0 . ausência; 1. pequena quantidade; 2 . média quantidade; 3 . grande quantidade de excretas com acúmulo no local. Foram considerados locais com pequena quantidade (escore 1) quando se observava excretas esparsas no ambiente com pouco material para coleta. Para média quantidade (escore 2) quando era observada quantidade considerável de excretas, mas sem acúmulo e para o escore 3 quando se tinha acúmulo, chegando a formar placas de excrementos.

Em cada local, com auxílio de espátulas, máscaras e luvas, foram colhidas entre 5 e 50 gramas de excretas, de acordo com o volume encontrado. $\mathrm{O}$ material foi acondicionado em frascos plásticos padronizados, em triplicata e identificados quanto à data e local da colheita, e mantidos em refrigeração por até 48 horas. Cada amostra foi homogeneizada utilizando-se $2 \mathrm{~g}$ de excretas em $20 \mathrm{ml}$ de solução salina acrescida de cloranfenicol $(0,2 \mathrm{~g} / \mathrm{l})$. Os tubos foram levados à agitação em vortex por 3 minutos, e mantidos em repouso por 30 minutos para decantação e obtenção do sobrenadante.

Uma alíquota de $0,1 \mathrm{ml}$ do sobrenadante foi retirada e semeada em triplicata, com alças de Drigalski estéreis, em ágar Sabouraud dextrose e em ágar Niger. Os cultivos foram incubados a $32^{\circ} \mathrm{C}$ e observados diariamente, por até dez dias, para avaliação macromorfológica das colônias. A micromorfologia foi realizada através do exame direto com tinta da China e lactofenol azul de algodão, visualizando-se em microscopia óptica (400X). Para confirmação do Cryptococcus neoformans, utilizou-se a prova da fenoloxidase (em meio ágar Niger), hidrólise da uréia, assimilação de fontes carbonadas e meio CGB (canavanina, glicina e azul de bromotimol).

\section{RESULTADOS E DISCUSSÃO}

Os isolados classificados como Cryptococcus neoformans mostraram, em meio ágar Sabouraud dextrose com cloranfenicol, colônias com aspecto superficial brilhante, liso, com consistência cremosa a mucóide, coloração branca a bege, que com o passar dos dias fluíam da superfície dos meios. No meio de ágar Niger, as colônias apresentaram coloração que variou do marrom claro ao marrom escuro. Microscopicamente, foram observadas células redondas ou ovais, envolvidas por uma cápsula de espessura variável, com ou sem brotamento. Na classificação bioquímica, foram positivos para a prova da fenoloxidase, hidrólise da uréia, assimilaram o Inositol, dextrose e dulcitol e na prova com meio CGB, não houve troca da coloração.
A quantidade de excretas encontradas durante a colheita foi muito variável (Tabela 1), sendo observado que em muitos locais era realizada a limpeza diária dos ambientes, não ocorrendo acúmulo de excretas, apesar da presença dos pombos. Dos 70 (100\%) locais selecionados para o estudo, observaram-se excretas de pombos em 26 $(37,1 \%)$ representados por prédios históricos $\left(n^{\circ}=8\right)$, torre de igreja $\left(n^{\circ}=1\right)$, engenhos e armazéns de arroz $\left(n^{\circ}=7\right)$, praça $\left(n^{\circ}=1\right)$ e locais ao ar livre $\left(n^{\circ}=9\right)$, sendo que o isolamento de Cryptococcus neoformans ocorreu em 26,9\% $\left(n^{\circ}=7 / 26\right)$ destes locais (Tabela 2).

TABELA 1 - Locais estudados e quantidade de excretas de pombos encontradas durante a coleta em áreas urbanas de Pelotas, RS.

\begin{tabular}{lccccc}
\hline & Número de & \multicolumn{4}{c}{ Volume de excretas } \\
\cline { 3 - 6 } Local & prédios e locais & 0 & 1 & 2 & 3 \\
\hline Torres de Igreja & 7 & 6 & - & 1 & - \\
Engenhos e armazéns de arroz & 7 & - & 2 & 3 & 2 \\
Praças & 14 & 13 & - & 1 & - \\
Prédios históricos & 33 & 25 & 2 & 3 & 3 \\
Locais ao ar livre & 9 & - & 8 & 1 & - \\
\hline Total & $\mathbf{7 0}$ & $\mathbf{4 4}$ & $\mathbf{1 2}$ & $\mathbf{9}$ & $\mathbf{5}$ \\
\hline
\end{tabular}

TABELA 2 - Percentual de isolamento de Cryptococcus neoformans em diferentes ambientes urbanos da Cidade de Pelotas, RS, em relação a presença de excretas e ao número de locais estudados.

\begin{tabular}{|c|c|c|c|c|c|c|}
\hline \multirow[b]{3}{*}{ Local } & \multirow{3}{*}{$\begin{array}{c}\text { Locais } \\
\text { visitados }\end{array}$} & \multirow{3}{*}{$\begin{array}{c}\text { Presença de } \\
\text { excretas }\end{array}$} & \multicolumn{4}{|c|}{ Isolamento } \\
\hline & & & \multicolumn{2}{|c|}{ relativo* } & \multicolumn{2}{|c|}{ absoluto $^{* *}$} \\
\hline & & & $\mathrm{n}^{\mathrm{o}}$ & $\%$ & $\mathrm{n}^{\mathrm{o}}$ & $\%$ \\
\hline Torres de Igreja & 7 & 1 & $1 / 1$ & 100 & $1 / 7$ & 14,3 \\
\hline Engenhos e armazéns de arroz & 7 & 7 & $2 / 7$ & 28,6 & $2 / 7$ & 28,6 \\
\hline Praças & 14 & 1 & $1 / 1$ & 100 & $1 / 14$ & 7,1 \\
\hline Prédios Históricos & 33 & 8 & $2 / 8$ & 25 & $2 / 33$ & 6 \\
\hline Locais ao ar livre & 9 & 9 & $1 / 9$ & 11 & $1 / 9$ & 11,1 \\
\hline Total & 70 & $26(37,1 \%)$ & $7 / 26$ & 26,9 & $7 / 70$ & 10 \\
\hline
\end{tabular}

*número de isolamentos/local com excretas, ${ }^{* *}$ número de isolamentos/total de locais estudados.

A maioria das igrejas visitadas tinha instalado barreiras mecânicas, como telas e tapumes em suas torres, a fim de evitar a entrada dos pombos. Somente em uma torre de igreja foi observada a presença de excretas, em quantidade média, de onde foi isolada a levedura, sendo esta igreja localizada no centro da cidade junto a uma praça arborizada, onde há grande oferta de alimento para as aves. O percentual de isolamento de Cryptococcus neoformans ficou em $14,3 \%$ em torres de igrejas $(1 / 7)$, entretanto se for considerada a presença de excretas $(1 / 1)$ o isolamento fica em $100 \%$. Baroni ${ }^{11}$ isolou Cryptococcus neoformans em $27 \%$ das amostras de excretas colhidas em dez torres de igrejas, segundo o autor o ambiente da torre de igreja apresentava temperatura amena sem incidência direta da luz solar, representando condições climáticas ideais para o desenvolvimento do fungo.

Dos engenhos e armazéns de arroz visitados, em sete foram encontradas excretas de pombos, recebendo escore que variou de 1 a 3 , sendo que os dois $(28,6 \%)$ locais que receberam escore 3 foi isolado o Cryptococcus neoformans. Durante a visitação foi observada grande quantidade de pombos nestes locais e, conseqüentemente, maior acúmulo de excretas, o que favoreceu o desenvolvimento do Cryptococcus neoformans. Segundo Levitz ${ }^{7}$, o acúmulo de excretas favorece o desenvolvimento de Cryptococcus neoformans que assimila a creatinina, ácido úrico, purinas e xantinas presentes nas excretas. 
Das 14 praças visitadas, somente em uma foi encontrada quantidade média de excretas, escore 2, de onde foi isolado o agente. Em outras nove amostras de locais ao ar livre, o isolamento de Cryptococcus neoformans só foi possível em uma (11,1\%). O isolado foi proveniente de excretas encontradas na área externa dos armazéns do porto. A praça encontra-se no centro de prédios históricos que servem de abrigo para os pombos, sendo muito arborizada, protegendo o material da radiação ultravioleta, da mesma maneira as excretas colhidas no porto estavam também protegidas por um semi-encoberto de telhas. As características climáticas locais, em que a umidade do ar é elevada e não ocorrem temperaturas extremas, podem ter possibilitado o isolamento do Cryptococcus neoformans nestes ambientes abertos. No Rio de Janeiro, onde ocorrem temperaturas mais altas, foi demonstrado o escasso isolamento desse agente em áreas ao ar livre, devido à ação da radiação solar, ação da chuva e vento, tornando escassa a quantidade de excretas e, conseqüentemente, dificultando o crescimento do fungo ${ }^{11}$.

Dos 33 prédios históricos, em oito $(24,2 \%)$ foram encontradas excretas, sendo que nos dois $(28,6 \%)$ que obtiveram escore 3 , foi isolado o Cryptococcus neoformans. Os sotãos e áreas de prédios históricos serviam de abrigo para os pombos, sendo que em alguns foi observado além de depósito de excretas, presença de penas, restos de ovos, pombos mortos em decomposição, associados a pouca ventilação, que servia para manutenção da viabilidade e multiplicação da levedura ${ }^{12}$. Em um dos prédios, havia grande quantidade de excretas frescas, sem acúmulo, já que a limpeza era feita diariamente, não ocorrendo o isolamento do Cryptococcus neoformans, o que coincide com observações de outros autores ${ }^{11,12}$ sendo argumentado que nas excretas frescas há uma proliferação bacteriana intensa, criando um $\mathrm{pH}$ alcalino que inibiria o crescimento da levedura.

Os sete isolados de Cryptococcus neoformans foram obtidos de locais que receberam escore 2 e 3 em relação a quantidade de excretas, representando assim, possíveis focos de risco para a população. $\mathrm{O}$ pombo doméstico ao contrário de outras aves, não retira o material fecal do ninho, permitindo que se formem pilhas de substrato no período de reprodução, favorecendo o desenvolvimento da levedura ${ }^{13,14}$. Outros autores têm descrito a presença do Cryptococcus neoformans em excretas de pombos em igrejas, ruas e praças, destacando o provável risco ao público, principalmente indivíduos imunocomprometidos que transitam por estes ambientes ${ }^{11,15}$.

A frequência (26,9\%) do Cryptococcus neoformans obtida foi semelhante às encontradas em outras regiões do Brasil, que variaram de 25 a 27\% ao serem pesquisadas em Porto Alegre, São Paulo e Rio de Janeiro ${ }^{11,15,16}$. Resultados inferiores para o isolamento de Cryptococcus neoformans foram descritos em Minas Gerais $(11,1 \%)^{17}$ e Santos, São Paulo $(13,9 \%)^{18}$. A diferença da frequência do Cryptococcus neoformans pode estar relacionada com o habitat ideal encontrado por estas aves em algumas cidades, que apresentam abrigos para construção de ninhos, disponibilidade de alimentos e ausência de predadores.

Baseado em dados de literatura e em nossos resultados, fica evidente que Cryptococcus neoformans está presente em locais onde ocorre acúmulo de excretas de pombos, e o estreito contato de humanos e animais com essas aves em áreas urbanas indica um risco em potencial para saúde pública, principalmente para indivíduos imunossuprimidos. O aumento da população de pombos ocorre devido a condições ideais de abrigo e alimentação, e são necessárias barreiras físicas em locais propícios para a construção de ninhos e formação de colônias desses animais, assim como controle de lixo e fontes que possam servir de alimentação para essas aves.

\section{CONFLITO DE INTERESSE}

Os autores declaram não haver nenhum tipo de conflito de interesse no desenvolvimento do estudo.

\section{SUPORTE FINANCEIRO}

\author{
CNPq, FAPERGS e CAPES.
}

\section{REFERÊNCIAS}

1. Kon AS, Grumach AS, Colombo AL, Penalva ACO, Wanke B, Telles FQ, et al. Consenso em criptococose. Rev Soc Bras Med Trop 2008; 41:524-544.

2. Kwon-Chung KJ, Bennett JE. Medical Mycology $1^{\text {st }}$ ed. Philadelphia: Lea \& Febiger; 1992. p. 397-446.

3. Kwon-Chung KJ, Boekhout T, Fell TJ, Diaz M. Proposal to conserve the name Cryptococcus gattii against C. hondurianus and C. bacillisporus (Basidiomycota, Hymenomycetes, Tremellomycetidae). Taxon 2002; 51:804-806.

4. Mitchell TG, Perfect JR. Cryptococcosis in the era of AIDS-100 years after the discovery of Cryptococcus neoformans. Clin Microbiol Rev 1995; 8:515-548.

5. Lazera MS, Salmito Cavalcanti MA, Londero AT, Trilles L, Nishikawa MM, Wanke B. Possible primary ecological niche of Cryptococcus neoformans. Med Mycol 2000; 38:379-383.

6. Cermeño JR, Hernández I, Cabello I, Orellán Y, Cermeño JJ, Albornoz R, et al. Cryptococcus neoformans and Histoplasma capsulatum in dove's (Columbia livia) excreta in Bolívar State, Venezuela. Rev Latinoam Microbiol 2006; 48:6-9

7. Levitz SM. The ecology of Cryptococcus neoformans and the epidemiology of cryptococcosis. Rev Infect Dis 1991; 13:1163-1169.

8. Rosario I, Acosta B, Colom F. La paloma y otras aves como reservorio de Cryptococcus spp. Rev Iberoam Micol 2008; 25:S13-S18.

9. Camargo HFA, Höfling E, Lencioni Neto F. Aves no Campus. $1^{\text {st }}$ ed. São Paulo: Editora da Universidade de São Paulo; 1993.

10. Regan AM, Metersky ML, Craven DE. Nosocomial dermatitis and pruritus caused by pigeon mite infestation. Arch Intern Med 1987; 147:2185-2187.

11. Baroni FA. Ocorrência de Cryptococcus neoformans em excretas de pombos localizadas em torres de igrejas na cidade do Rio de Janeiro: fatores de virulência e sensibilidade aos antifúngicos [Tese de Doutorado]. [São Paulo(SP)]: Instituto de Ciências Biomédicas da Universidade de São Paulo; 2001.

12. Civila E, Conti-Diaz IA. Aislamiento de Cryptococcus neoformans de excretas secas de palomas en la ciudad de Montevideo. Rev Urug Pat Clin Microbiol 1976; 3:41-48.

13. Caicedo LD, Alvarez MI, Llanos CE, Molina D. Cryptococcus neoformans em excretas de palomas del perímetro urbano de Cali. Colomb Med 1996; 27:106109.

14. Emmons CW. Saprophytic sources of Cryptococcus neoformans associated with the pigeon (Columba livia). Am J Trop Med Hyg 1955; 62:227-232.

15. Macêdo RCL, Santos MJS, Oliveira AM, Oliveira MRF, Lopes SP, Ferreira F, et al. Cryptococcus neoformans em ambientes públicos na cidade do Rio de Janeiro. Dados preliminares. In: Anais do III Congresso Brasileiro de Micologia; Águas de Lindóia, SP; 2001.

16. Montenegro H, Paula CR. Environmental isolation of Cryptococcus neoformans var gattii and Cryptococcus neoformans var. neoformans in the city of São Paulo, Brazil. Med Mycol 2000; 38:385-390.

17. Rezende DG, Siqueira AM. Ocorrência de Cryptococcus neoformans em fezes de aves na cidade de Alfenas, MG. In: Anais do III Congresso Brasileiro de Micologia; Águas de Lindóia, SP; 2001.

18. Soares MCB, Paula CR, Dias ALT, Caseiro MM, Costa SOP Environmental strains of Cryptococcus neoformans variety grubii in the city of Santos, SP, Brasil. Rev Inst Med Trop São Paulo 2005; 47:31-36. 